\title{
Is Nature Fundamentally Continuous or Discrete, and How Can These Two Different But Very Useful Conceptions Be Fully Reconciled?
}

\author{
Andrew Walcott Beckwith \\ Physics Department, College of Physics, Chongqing University, Chongqing, China \\ Email: Rwill9955b@gmail.com, abeckwith@uh.edu
}

How to cite this paper: Beckwith, A.W. (2018) Is Nature Fundamentally Continuous or Discrete, and How Can These Two Different But Very Useful Conceptions Be Fully Reconciled? Journal of High Energy Physics, Gravitation and Cosmology, 4, 796-813.

https://doi.org/10.4236/jhepgc.2018.44045

Received: April 29, 2018

Accepted: October 28, 2018

Published: October 31, 2018

Copyright $\odot 2018$ by author and Scientific Research Publishing Inc. This work is licensed under the Creative Commons Attribution International License (CC BY 4.0).

http://creativecommons.org/licenses/by/4.0/

\begin{abstract}
Our contention is that reality is actually analog, but at a critical limit, when the Octonian gravity condition kicks in, for a time it is made to appear discrete. This is due to an initial phase transition just at the start of the big bang. Our second consideration is that symmetry breaking models, i.e. the Higgs boson, are in themselves not appropriate or necessary for the formation of particles with mass just before Octonionic gravity which could arise in pre-Planckian physics models without a potential. Finally, the necessity of potentials for pre-Octonionic gravity physics can be circumvented via judicious use of Scherrer k essence physics.
\end{abstract}

\section{Keywords}

Continuous and Discrete Space-Time, Octonionic Geometry, Analog Physics

\section{Introduction}

Our presentation takes note of several developments. First of all a feed into cosmological vacuum energy has been modeled, and we have ideas as to how to interrelate four and five dimensional vacuum energies. Secondly, a mechanism for the onset of Octonian gravity is stated, as a consequence as to a buildup of a peak temperature for its inception, and at the time space time flattens. The onset of pre-Octonionic gravity, with tiny masses associated with gravitons, is in line with Quantum mechanics as embedded within a larger, non-linear classical theory (i.e. go to the Pilot Model, to get an idea of what is involved, plus t'Hoofts deterministic quantum mechanics construction). Thirdly, we suggest that the transition from highly curved space time, which is pre-Octonian gravity, i.e. Non-quantum state, to quantum state, is due to a chaotic mapping which we 
present in this document. The chaotic mapping also has that there would be an explosion of the degrees of freedom up to Planckian space time. i.e. this degree of freedom explosion would be where we obtain quantum dynamics. Thermal inputs for the push to quantum dynamics are the first topic brought up for our perusal of this document.

\section{Vacuum Energy, Sources and Commentary}

Begin first with looking at different value of the cosmological vacuum energy parameters, in four and five dimensions [1]

$$
\left|\Lambda_{5-\operatorname{dim}}\right| \approx c_{1} \cdot\left(1 / T^{\alpha}\right)
$$

in contrast with the more traditional four-dimensional version of the same, minus the minus sign of the Brane world theory version. The five-dimensional version is actually connected with Brane theory and higher dimensions, whereas the four-dimensional version is linked to more traditional De Sitter space-time geometry, as given by Park (2003) [2]

$$
\Lambda_{4-\operatorname{dim}} \approx c_{2} \cdot T^{\beta}
$$

If one looks at the range of allowed upper bounds of the cosmological constant, the difference between what Barvinsky (2006) [3] recently predicted, and Park (2003) [2] is:

$$
\Lambda_{4-\operatorname{dim}} \propto c_{2} \cdot T^{\beta} \underset{\text { graviton-production-as-time }>(\text { Planck })}{\longrightarrow} 360 \cdot m_{P}^{2} \ll c_{2} \cdot\left[T \approx 10^{32} \mathrm{~K}\right]^{\beta}
$$

Right after the gravitons are released, one still sees a drop-off of temperature contributions to the cosmological constant. Then one can write, for small time values $t \approx \delta^{1} \cdot t_{P}, \quad 0<\delta^{1} \leq 1$ and for temperatures sharply lower than $T \approx 10^{12}$ Kelvin, Beckwith (2007), where for a positive integer $n[4]$

$$
\frac{\Lambda_{4-\operatorname{dim}}}{\left|\Lambda_{5-\text { dim }}\right|}-1 \approx \frac{1}{n}
$$

If there is an order of magnitude equivalence between such representations, there is a quantum regime of gravity that is consistent with fluctuations in energy and growth of entropy. An order-of-magnitude estimate will be used to present what the value of the vacuum energy should be in the neighborhood of Planck time in the advent of nucleation of a new universe. The significance of Equation (4) is that at very high temperatures, it re enforces what the author brought up with Tigran Tchrakian, in Bremen [5], August 29 $9^{\text {th }}$, 2008. i.e., one would like to have a uniform value of the cosmological constant in the gravitating Yang-Mills fields in quantum gravity in order to keep the gauges associated with instantons from changing. When one has, especially for times $t_{1}, t_{2}<$ Planck time $t_{P}$ and $t_{1} \neq t_{2}$, with temperature $T\left(t_{1}\right) \neq T\left(t_{2}\right)$, then

$\Lambda_{4}\left(t_{1}\right) \neq \Lambda_{4}\left(t_{2}\right)$. i.e., in the regime of high temperatures, one has $T\left(t_{1}\right) \neq T\left(t_{2}\right)$ for times $t_{1}, t_{2}<$ Planck time $t_{P}$ and $t_{1} \neq t_{2}$, such that gauge invariance necessary for soliton (instanton) stability is broken [5]. That breaking of instanton stability due to changes of $\Lambda_{4}\left(t_{1}\right) \neq \Lambda_{4}\left(t_{2}\right)$ will be our point of where we move 
from an embedding of quantum mechanics in an analog reality, to the quantum regime. I.e. as one reaches to high temperature, analog reality mimics digital quantum mechanics. Let us now look at different characterizations of the discontinuity, which is the boundary between analog reality, and Octonian gravity. First of all, one can look at scale factor evolution.

\section{What Leads to Causal Discontinuity in Scale Factor Evolution?}

The Friedmann equation [6] for the evolution of a scale factor $a(t)$,

$$
(\dot{a} / a)^{2}=\frac{8 \pi G}{3} \cdot\left[\rho_{\text {rel }}+\rho_{\text {matter }}\right]+\frac{\Lambda}{3}
$$

suggests a non-partially ordered set evolution of the scale factor with evolving time, thereby implying a causal discontinuity. The validity of this formalism is established by rewriting the Friedman equation as follows: $a\left(t^{*}\right)<l_{P}$ for $t^{*}<t_{P}=$ Planck time, and $a_{0} \equiv l_{P}$, for a discrete equation model of Equation (6) $[4]$

$$
\begin{aligned}
& {\left[\frac{a\left(t^{*}+\delta t\right)}{a\left(t^{*}\right)}\right]-1} \\
& <\frac{\left(\delta t \cdot l_{P}\right)}{(\sqrt{3 / 8 \pi \Lambda})} \cdot\left[\frac{1}{24 \pi \cdot a^{2}\left(t^{*}\right)}+\frac{1}{\Lambda} \cdot\left[\left(\rho_{r e l}\right)_{0} \cdot \frac{a_{0}^{4}}{a^{6}\left(t^{*}\right)}+\left(\rho_{m}\right)_{0} \cdot \frac{a_{0}^{3}}{a^{5}\left(t^{*}\right)}\right]\right]^{1 / 2} \\
& \underset{\delta t \rightarrow \varepsilon^{+}, \Lambda \neq \infty, a \neq 0}{\longrightarrow}\left(\frac{\delta t \cdot\left[l_{P} / a\left(t^{*}\right)\right]}{\sqrt{3 / 8 \pi}}\right) \cdot \sqrt{\frac{\left(\rho_{r e l}\right)_{0} a_{0}^{4}}{a^{4}\left(t^{*}\right)}+\frac{\left(\rho_{m}\right)_{0} a_{0}^{3}}{a^{3}\left(t^{*}\right)}} \approx \varepsilon^{+} \ll
\end{aligned}
$$

So in the initial phases of the big bang, with very large vacuum energy $\neq \infty$ and $a\left(t^{*}\right) \neq 0,0<a\left(t^{*}\right) \ll 1$, the following relation, which violates (signal) causality, is obtained for very small fluctuation $a\left(t^{*}\right)<l_{P}$ for $t^{*}<t_{P}=$ Planck time, and $a_{0} \neq l_{P}, a_{0} \gg l_{P}$, which indicates that [6]

$$
\rho_{\text {rel }} \equiv\left(\frac{a_{\text {present-era }}}{a(t)}\right)^{4} \cdot\left(\rho_{\text {rel }}\right)_{\text {present-era }}
$$

and

$$
\rho_{m} \equiv\left(\frac{a_{\text {present-era }}}{a(t)}\right)^{3} \cdot\left(\rho_{m}\right)_{\text {present-era }}
$$

Using the above equation creates the following as plausible estimates, which can be reviewed, as needed. For large, but not infinite temperatures, and for $\Lambda \sim c_{1} T^{\alpha}$

$$
\left(\frac{\delta t \cdot\left[l_{P} / a\left(t^{*}\right)\right]}{\sqrt{3 / 8 \pi}}\right) \cdot \sqrt{\frac{\left(\rho_{r e l}\right)_{0} a_{0}^{4}}{a^{4}\left(t^{*}\right)}+\frac{\left(\rho_{m}\right)_{0} a_{0}^{3}}{a^{3}\left(t^{*}\right)}} \sim 10^{-45} \cdot 10^{1} \cdot \sqrt{10^{80}} \approx 10^{-4} \ll 1
$$

If we examine what happens with $\left|\Lambda_{5-\operatorname{dim}}\right| \sim c_{2} T^{-\beta}$. 
For times $t>t_{P} \rightarrow$ today, a stable instanton is assumed, along the lines brought up by t'Hooft [7], due to the stable $\Lambda_{4-\text { dim }} \approx$ constant $\sim$ very small value, roughly at the value given today. This assumes a radical drop-off of the cosmological constant for, say right after the electroweak transition. This would be in line with Kolb's assertion of the net degrees of freedom in space-time drop from about 1000 to less than two, especially if $t>t_{P} \rightarrow$ today in terms of the value of time after the big bang. The supposition we are making here is that the value of $N$ so obtained is actually proportional to a numerical graviton density we will refer to as $\langle n\rangle$, provided that there is a bias toward HFGW, which would mandate a very small value for $V \approx R_{H}^{3} \approx \lambda^{3}$. Furthermore, structure formation arguments, as given by Perkins [8] give ample evidence that if we use an energy scale, $m$, over a Planck mass value $M_{\text {Planck }}$, as well as contributions from field amplitude $\phi$, and using the contribution of scale factor behavior $\frac{\dot{a}}{a} \equiv H \approx-m \cdot \frac{\phi}{3 \cdot \dot{\phi}}$, where we assume $\ddot{\phi} \cong 0$ due to inflation

$$
\frac{\Delta \rho}{\rho} \sim H \Delta t \sim \frac{H^{2}}{\dot{\phi}} \sim\left(\frac{m}{M_{\text {Planck }}}\right) \times\left(\frac{\phi}{M_{\text {Planck }}}\right) \sim 10^{-5}
$$

At the very onset of inflation, $\phi \ll M_{\text {Planck }}$, and if $m$ (assuming $\hbar=c=1$ ) is due to inputs from a prior universe, we have a wide range of parameter space as to ascertain where $\Delta S \approx \Delta N_{\text {gravitons }} \neq 10^{88}$ comes from and plays a role as to the development of entropy in cosmological evolution In the next Chapter, we will discuss if or not it is feasible/reasonable to have data compression of prior universe "information". It suffices to say that if $S_{\text {initial }} \sim 10^{5}$ is transferred from a prior universe to our own universe at the onset of inflation,, at times less than Planck time $t_{P} \sim 10^{-44}$ seconds, that enough information MAY exit for the preservation of the prior universe's cosmological constants, i.e. $\hbar, G, \alpha$ (fine structure constant) and the like. We do not have a reference for this and this supposition is being presented for the first time. Times after $t=10^{\wedge}-44$ are not less important. But that the "constant's memory" is already imprinted in the universe, so to speak, i.e. a memory transfer is implied as far as being transferred from the beginning. Confirmation of this hypothesis depends upon models of how much "information" $\hbar, G, \alpha$ actually require to be set in place, at the onset of our universe's inflation, a topic which we currently have no experimental way of testing at this current time.

\section{Is Each "Particle Count Unit" as Brought Up by Ng, Is Equivalent to a Brane-Anti Brane "Unit in Brane Treatments of Entropy"? How Does This Tie in with String/Brane Theory Treatments of Entropy?}

It is useful to state this convention for analyzing the resulting entropy calculations, because it is a way to explain how and why the number of instanton-anti instanton pairs, and their formulation and break up can be linked to the growth of entropy as given by infinite quantum statistics as given in [9] where we use a 
graviton count, $\mathrm{N}$, so as to give initial entropy. If, as an example, there is a linkage between quantum energy level components of the quantum gas as brought up by Glinka [10] (2007), and the number of instanton-anti instanton pairs, then it is possible to ascertain a linkage between a Wheeler De Witt worm hole introduction of vacuum energy [11] from a prior universe to our present universe, and the resulting brane-anti brane (instanton-anti instanton) units of entropy. What we should keep in mind, is that, as given by W. D. Goldberger, [12] that even here, the traceless moment

$$
Q^{i j}=\sum_{a} m_{a} \cdot\left[x_{a}^{i} x_{a}^{j}-\frac{1}{3} \cdot x_{a}^{2} \delta_{i j}\right]
$$

is used. As a source of gravitational waves. The end result is that even here, in relic conditions the output power is of the form, with the dots being derivatives in time, and \langle\rangle referring to a time averaged quantity

$$
P=\frac{G_{N}}{5} \cdot\left\langle\dddot{Q}_{i j} \dddot{Q}_{i j}\right\rangle
$$

We can prove that graviton gas emerges from the quantum vacuum for a special case: with enough random quantum vacuum "motion" there will be quadrupole moments in distribution changes, resulting in gravitons. i.e. the relic inflationary physics conditions assure this sort of framing of the problem. This is a counter part as to what Asakawa et al. [12], (2001), and [13] (2006) suggested for quark gluon gases, and the 2nd order phase transition written up by Torrieri et al. [14] (2008) brought up at the nuclear physics Erice (2008) school, in discussions with the author. Furthermore, finding out if or not it is either a drop in viscosity [13], and especially [14] when $\left|\frac{\eta}{s} \approx \varepsilon^{+}\right| \ll \frac{1}{4 \pi}$, or a major increase in entropy density may tell us how much information is, indeed, transferred from a prior universe to our present. If it is $s \rightarrow \infty$, for all effective purposes, at the moment after the pre big bang configuration, likely then there will be a high degree of "information" from a prior universe exchanged to our present universe. If on the other hand, $\eta \rightarrow 0^{+}$due to restriction of "information" from four dimensional "geometry" to a variable fifth dimension, so as to indicate almost infinite collisions with a closure of a fourth dimensional "portal" for information flow, we are then invoking [10] and Glinkas quantum gas model. In doing so, we would then, in the strict application of [10] have conditions leading to almost no information exchange. In physical cases where [10] is not used but instead we have ideas similar to a quark-gluon plasmal, we would have to utilize the physics of cases similar to [11], [12], in place of a strict graviton gas as cited in [10].

\section{Consider Now What Could Happen with a Phenomenological Model Bases upon the Following Inflection Point i.e. Split Regime of Different Potential Behavior}

$$
V(\phi)=g \cdot \phi^{\alpha}
$$


De facto, what we come up with pre, and post Planckian space time regimes, when looking at consistency of the emergent structure is the following. Namely,

$$
V(\varphi) \propto \phi^{|\alpha|} \text { for } t<t_{\text {PLanck }}
$$

Also, we would have

$$
V(\varphi) \propto 1 / \phi^{|\alpha|} \text { for } t \gg t_{\text {PLanck }}
$$

The switch between Equation (14a) and Equation (14b) is not justified analytically. i.e. it breaks down. Beckwith et al. (2011) designated this as the boundary of a causal discontinuity [4]. Now according to Weinberg [15], if $\in=\frac{\lambda^{2}}{16 \pi G}, H=1 / \in t$ so that one has a scale factor behaving as

$$
a(t) \propto t^{1 / \epsilon}
$$

Then, if

$$
|V(\phi)| \ll(4 \pi G)^{-2}
$$

there are no quantum gravity effects worth speaking of. i.e., if one uses an exponential potential a scalar field could take the value of, when there is a drop in a field from $\phi_{1}$ to $\phi_{2}$ for flat space geometry and times $t_{1}$ to $t_{2}$ [15]

$$
\phi(t)=\frac{1}{\lambda} \ln \left[\frac{8 \pi G g \in^{2} t^{2}}{3}\right]
$$

Then the scale factors, from Planckian time scale as [14]

$$
\frac{a\left(t_{2}\right)}{a\left(t_{1}\right)}=\left(\frac{t_{2}}{t_{1}}\right)^{1 / \epsilon}=\exp \left[\frac{\left(\phi_{2}-\phi_{1}\right) \lambda}{2 \in}\right]
$$

The more $\frac{a\left(t_{2}\right)}{a\left(t_{1}\right)} \gg 1$, then the less likely there is a tie in with quantum gravity. Note those that the way this potential is defined is for a flat, Roberson-Walker geometry, and that if and when $t_{1}<t_{\text {Planck }}$ then what is done in Equation (11) no longer applies, and that one is no longer having any connection with even an octonionic Gravity regime. If so, as indicated by Beckwith, et al. (2011) [16] one may have to tie in graviton production due to photonic ("light") inputs from a prior universe, i.e. a causal discontinuity, with consequences which will show in both GW and graviton production. This leads to Planck's mass $m_{\text {Plank }} \sim 2.17645 \times 10^{-35}$ kilograms, as alluded to by Barvinsky [3].

$$
\Lambda_{4-\mathrm{Dim}} \propto c_{2} T_{\text {Temp }}^{\beta} \underset{\text { Graviton-Production }}{\longrightarrow} 360 \cdot m_{\text {Planck }}^{2} \ll c_{2}\left[T \cong 10^{12} \text { Kelvin }\right]_{\text {Temp }}^{\beta}
$$

Right after the gravitons are released, there is still a drop off of temperature contributions to the cosmological constant. For a small time value, $t \equiv \delta^{1} t_{\text {Planck }}$, where $0<\delta^{1} \leq 1$ and for temperatures sharply lower than $=10$ to the 32 nd power Kelvin, this difference is the ratio of the value of the four-dimensional version of the cosmological constant divided by the absolute value of the five 
dimensional cosmological constant, which is equal to 1 plus $1 / n$, where $n$ is a positive integer. The transition outlined in Equation (12) above has a starting point with extremely high temperatures given by a vacuum energy transferal between a prior universe and our present universe. We will next then, look at this model for a possible increase in the degrees of freedom, initially now we should consider what constitutes a thermal change which may impact the physics of the change as indicated from Equation (14a) to Equation (14b).

\section{Increase in Degrees of Freedom in the Sub Planckian Regime}

Starting with [17]

$$
E_{\text {Thermal }} \cong \frac{k_{B} \cdot T_{\text {Temp }}}{2} \propto\left[\Omega_{0} \breve{T}\right] \sim \tilde{\beta}
$$

The assumption is that there would be an initial fixed entropy arising, with $\bar{N}$ as a nucleated structure arising in a short time interval as a temperature $T_{\text {Temp }} \in\left(0^{+}, 10^{19} \mathrm{GeV}\right)$ arrives. One then obtains, dimensionally speaking [17],

$$
\frac{\Delta \tilde{\beta}}{\text { dist }} \cong \frac{5 k_{B} \cdot T_{\text {Temp }}}{2} \cdot \frac{\bar{N}}{\text { dist }} \sim q E_{\text {Net-electric-field }} \sim\left[T_{\text {Temp }} \Delta S / \text { dist }\right]
$$

The parameter, as given by $\Delta \tilde{\beta}$ will be one of the parameters used to define chaotic Gaussian mappings. Candidates as to the inflation potential would be in powers of the inflation, I.e. in terms of $\phi^{N}$, with $N=4$ effectively ruled out, and perhaps $N=2$ an admissible candidate (chaotic inflation). For $N=2$, one gets [17]

$$
\Delta S \equiv\left[\frac{\hbar}{T_{\text {Temp }}}\right] \cdot \sqrt{2 k+\frac{3}{\eta^{2} \phi^{2}}} \sim n_{\text {Particle-count }}
$$

If the inputs into the inflation, as given by $\phi$ becomes from Equation (6) a random influx of thermal energy from temperature, we will see the particle count on the right hand side of Equation (15) above a partly random creation of $n_{\text {Particle-count }}$ which we claim has its counterpart in the following treatment of an increase in degrees of freedom. The way to introduce the expansion of the degrees of freedom from nearly zero, at the maximum point of contraction to having $N(T) \sim 10^{3}$ is to first define the classical and quantum regimes of gravity in such a way as to minimize the point of the bifurcation diagram affected by quantum processes [17]. If we suppose smoothness of space time structure down to a grid size of $l_{\text {Plank }} \sim 10^{-35}$ centimeters at the start of inflationary expansion we have when doing this construction what would be needed to look at the maximum point of contraction, setting at $l_{\text {Plank }} \sim 10^{-35}$ centimeters the quantum "dot" or infometron, as a de facto measure zero set, as the bounce point, with classical physics behavior before and after the bounce "through" the quantum dot. Dynamical systems modeling could be directly employed right "after" evolution through the "quantum dot" regime, with a transfer of crunched in 
energy to Helmholtz free energy, as the driver "force" for a Gauss map type chaotic diagram right after the transition to the quantum "dot" point of maximum contraction. The diagram, in a bifurcation sense would look like an application of the Gauss mapping of [17].

$$
x_{i+1}=\exp \left(-\tilde{\alpha} \cdot x_{i}^{2}\right)+\tilde{\beta}
$$

In dynamical systems type parlance, one would achieve a diagram, with tree structure looking like what was given by Binous [18], using the material cited as given by Binous material written up by Lynch [18]. Now that we have a model as to what could be a change in space time geometry, let us consider what may happen during the Higgs mechanism and why it may not apply as expected in very early universe geometry. In particular we wish to cite the work given by Sarkar, in [19] as given, namely:

\section{Higgs Mechanism, and Its Consequence in the Onset of Inflation. i.e. Why It Could Break Down}

Let us begin first with a $\mathrm{U}(1)$ gauge theory, the Fermion $\psi$ would transform locally as given by

$$
\psi \rightarrow \psi^{\prime}=(\exp [-i g \vartheta \cdot q(x)]) \cdot \psi
$$

This has a Lagrangian given by, an expression for covariant derivative $D_{\mu}=\partial_{\mu}+i g \vartheta A_{\mu}(x)$, and also $F_{\mu \nu}=\partial_{\mu} A_{v}-\partial_{\nu} A_{\mu}$, so that $\partial_{\mu} F_{\mu \nu}=j_{v}$ for current. With the mass term for the gauge boson $A_{\mu}$ not allowed by gauge symmetry

$$
\varsigma=i \bar{\psi} \gamma^{\mu} D_{\mu} \psi+m \bar{\psi} \psi-\frac{1}{4} F^{\mu v} F_{\mu v}
$$

A way to allow for the mass to be factored in, i.e. look at $\phi \rightarrow \phi^{\prime}=(\exp [-i g \vartheta \cdot q(x)]) \cdot \phi$, and then

$$
\varsigma(\phi)=i D^{\sigma} \phi^{+} D_{\mu} \phi-\frac{1}{2} \mu^{2} \phi^{+} \phi-\frac{1}{4} \lambda\left(\phi^{+} \phi\right)^{2}
$$

If $\mu^{2}<0$, the potential has a minimum, with $\left\langle\phi^{+} \phi\right\rangle=v^{2}=-\mu^{2} / \lambda>0$, with a $\mathrm{VeV}\langle\phi\rangle=v$. Then

$$
\phi=(\eta+v) \exp [i \sigma / v]
$$

As stated by U. Sarkar, a kinetic energy term for the scalar field, namely $g^{2} v^{2} A^{\mu} A_{\mu} \subset D^{\mu} \phi^{+} D_{\mu} \phi$ is such that a mass term may exist. Now as to why it is stated that this procedure may break down. A scalar field will no longer be massless if the following step is taken, namely an explicit symmetry breaking term $m^{2}\left(\phi \phi+\phi^{*} \phi^{*}\right)$ will allow a scalar field $\phi$ to be expanded about a $\mathrm{VeV}$ $\langle\phi\rangle=v$ with

$$
\phi=(\eta+v) \exp [i \sigma / v] \sim \eta+v+i \sigma-\sigma^{2} / 2 v
$$

so that the mass of $\sigma$ is $m^{2}$, so $\sigma$ is a pseudo nambu goldstone boson. If 
one wishes to have explicit examples of the VeVs, then consider

$$
\begin{aligned}
S U(5) \rightarrow S U(4) \times U(1) \Rightarrow\langle\phi\rangle & =\left(\begin{array}{ccccc}
1 & 0 & 0 & 0 & 0 \\
0 & 1 & 0 & 0 & 0 \\
0 & 0 & 1 & 0 & 0 \\
0 & 0 & 0 & 1 & 0 \\
0 & 0 & 0 & 0 & -4
\end{array}\right) \\
S U(5) \rightarrow S U(3) \times S U(2) \times U(1) \Rightarrow\langle\phi\rangle & =\left(\begin{array}{ccccc}
1 & 0 & 0 & 0 & 0 \\
0 & 1 & 0 & 0 & 0 \\
0 & 0 & 1 & 0 & 0 \\
0 & 0 & 0 & -3 / 2 & 0 \\
0 & 0 & 0 & 0 & -3 / 2
\end{array}\right)
\end{aligned}
$$

In the case of when one is looking at when the $\mathrm{VeV}$ is congruent with a broken symmetry potential, as of the form $m^{2}\left(\phi \phi+\phi^{*} \phi^{*}\right)$, which no longer exists in the situation where one is looking at $\mathrm{k}$ essence inflation, we then will be having to consider the situation is given by: The main point as to why the Higgs paradigm may break down lies in the fact that emergent structure can be formulated without use of a broken symmetry potential as given by $m^{2}\left(\phi \phi+\phi^{*} \phi^{*}\right)$.

\section{How to Have Particle Formation without a Broken Symmetry Potential. Use of Scherrer $k$ Esesence}

In particular, the situation to watch can be diagrammed out by appendix entry where we are looking at the $k$ essence scenario as in [20] and [21]. This means we have a small value for the "growth of density perturbations" along the lines of [20]

$$
C_{S}^{2} \cong \frac{1}{1+2 \cdot\left(X_{0}+\tilde{\varepsilon}_{0}\right) \cdot\left(1 / \tilde{\varepsilon}_{0}\right)} \equiv \frac{1}{1+2 \cdot\left(1+\frac{X_{0}}{\tilde{\varepsilon}_{0}}\right)}
$$

if we can approximate the kinetic energy from

$$
\left(\partial_{\mu} \phi\right) \cdot\left(\partial^{\mu} \phi\right) \equiv\left(\frac{1}{c} \cdot \frac{\partial \phi}{\partial \cdot t}\right)^{2}-(\nabla \phi)^{2} \cong-(\nabla \phi)^{2} \rightarrow-\left(\frac{\mathrm{d}}{\mathrm{d} x} \phi\right)^{2}
$$

if we assume that we are working with a comparatively small contribution w.r.t. time variation, but a very large in many cases contribution w.r.t. spatial variation of phase

$$
\begin{gathered}
\left|X_{0}\right| \approx \frac{1}{2} \cdot\left(\frac{\partial \phi}{\partial x}\right)^{2} \gg \tilde{\varepsilon}_{0} \\
0 \leq C_{S}^{2} \approx \varepsilon^{+} \ll 1
\end{gathered}
$$

and

$$
w \equiv \frac{p}{\rho} \cong \frac{-1}{1-4 \cdot\left(X_{0}+\tilde{\varepsilon}_{0}\right) \cdot\left(\frac{F_{2}}{F_{0}+F_{2} \cdot\left(\tilde{\varepsilon}_{0}\right)^{2}} \cdot \tilde{\varepsilon}_{0}\right)} \approx 0
$$


We get these values for the phase being nearly a "box" of height approximately scaled to be about $2 \cdot \pi$ and of width $L$. Which we obtained by setting

$$
\phi \approx \pi \cdot[\tanh b \cdot(x+L / 2)-\tanh b \cdot(x-L / 2)]
$$

This means that the initial conditions we are hypothesizing are in line with the equation of state conditions appropriate for a cosmological constant but near zero effective sound speed. As it is, we approximate.

This is occurring in the regime in which Octonian gravity initially does not apply and which eventually it does apply. So, let us look at the following

\section{Relevance to Octonian Quantum Gravity Constructions? Where Does Non-Commutative Geometry Come into Play?}

Crowell [22] wrote on page 309 that in his Equation (8.141), namely

$$
\left[x_{j}, p_{k}\right] \cong-\beta \cdot\left(l_{\text {Planck }} / l\right) \cdot \hbar \cdot T_{i j k} x_{k} \underset{\text { Graviton-Production }}{\longrightarrow} i \hbar \delta_{i j}
$$

Here, $\beta$ is a scaling factor, while we have, above, after a certain spatial distance, a Kroniker function so that at a small distance from the confines of Planck time, we recover our quantum mechanical behavior. Our contention is, that since Equation (35) depends upon energy-momentum being conserved as an average about quantum fluctuations, that if energy-momentum is violated, in part, that Equation (35) falls apart. How Crowell forms Equation (35) at the Planck scale depends heavily upon energy-Momentum being conserved [1]. Our construction VIOLATES energy-momentum conservation. N. Poplawski [23] also has a very revealing construction for the vacuum energy, and cosmological constant which we reproduce, here

$\Lambda=\left[\frac{3 \kappa^{2}}{16}\right] \cdot\left(\bar{\psi} \gamma_{j} \cdot \gamma^{5} \psi\right) \cdot\left(\bar{\psi} \gamma_{j} \cdot \gamma^{5} \psi\right)$ and $\rho_{\Lambda}=\left[\frac{3 \kappa}{16}\right] \cdot\left(\bar{\psi} \gamma_{j} \cdot \gamma^{5} \psi\right) \cdot\left(\bar{\psi} \gamma_{j} \cdot \gamma^{5} \psi\right)(36)$

Poplawski writes that formation of the above, is:

"Such a torsion-induced cosmological constant depends on spinor fields, so it is not constant in time (it is constant in space at cosmological scales in a homogeneous and isotropic universe). However, if these fields can form a condensate then the vacuum expectation value of (Equation (37)) will behave like a real cosmological constant."

Poplawski [23] writes his formulation in terms of a quark-gluon QCD based condensate. Our contention is that once a QCD style condensate breaks up there will afterwards be NO equivalent structure to Equation (35) and Equation (36) even at the beginning of inflation right after the break down of space time particle transfer. Once that condensate structure is not possible then as quantified by Equation (8.140) of Crowell [22], the following will not hold:

$$
\oint p_{i} \mathrm{~d} x_{k}=\hbar \delta_{i k}
$$

Equation (8.40) of the Crowell manuscript also makes the additional assumption, that non flat space has a geometric non-commutativity protocol which is delineated by the following spatial relationship. When Equation (38) goes to ze- 
ro, we recover the regime in which quantum mechanics holds.

$$
\left[x_{j}, x_{k}\right]=\beta \cdot l_{\text {Planck }} \cdot \hbar \cdot T_{j k i} x_{i}
$$

Does the (QCD) condensate occur post plankian, and not work for pre plankian regime? Yes. The problem lies with Equation (8.140) of Crowell [22] with the final equality not holding. If one were integrating across a causal barrier,

$$
\oint\left[x_{j}, p_{i}\right] \cdot \mathrm{d} x_{k} \cong-\oint p_{i} \cdot\left[x_{j}, \mathrm{~d} x_{k}\right]=\beta \cdot l_{\text {Planck }} \cdot T_{j k i} x_{k} \cdot \oint p_{i} \mathrm{~d} x_{j} \neq \hbar \beta \cdot l_{\text {Planck }} \cdot T_{j k i}
$$

Very likely, across a causal boundary, between $\pm l_{\text {Planck }}$ across the boundary due to the causal barrier, one would have

$$
\oint p_{i} \mathrm{~d} x_{j} \neq \hbar \cdot \delta_{j k} \oint p_{i} \mathrm{~d} x_{k}=0
$$

i.e.

$$
\left.\oint_{ \pm l_{\text {Planck }}} p_{i} \mathrm{~d} x_{k}\right|_{i=k} \rightarrow 0
$$

If so, then [1]

$$
\left[x_{j}, p_{k}\right] \neq-\beta \cdot\left(l_{\text {Planck }} / l\right) \cdot \hbar \cdot T_{i j k} x_{k} \text { and does not } \rightarrow i \hbar \delta_{i j}
$$

Equation (42) in itself would mean that in the pre Planckian physics regime, and in between $\pm l_{\text {Planck }}$, QM no longer applies. What we will do next is to begin the process of determining a regime in which Equation (42) may no longer hold via experimental data sets. As an example of present confusion, please consider the following discussion where leading cosmologists, i.e. Sean Carroll [24] (2005) asserted that there is a distinct possibility that mega black holes in the center of spiral galaxies have more entropy, in a calculated sense, i.e. up to $10^{90}$ in non dimensional units. This has to be compared to Carroll's (2005) [24] stated value of up to $10^{88}$ in non dimensional units for observable non dimensional entropy units for the observable universe. Assume that there are over one billion spiral galaxies, with massive black holes in their center, each with entropy $10^{90}$, and then there is due to spiral galaxy entropy contributions $10^{6} \times 10^{90}=10^{96}$ entropy units to contend with, vs. $10^{88}$ entropy units to contend with for the observed universe, i.e. at least a ten to the eight order difference in entropy magnitude to contend with. A further datum to consider is that Equation (42) with its variance of density fluctuations may eventually be linkable to Kolmogrov theory as far as structure formation. If we look at R. M. S. Rosa [25] [26] (2006), and energy cascades of the form of the "energy dissipation law", assuming $u_{0}, l_{0}$ are minimum velocity and length, with velocity less than the speed of light, and the length at least as large, up to $10^{6}$ time larger than Planck length $l_{\text {Planck }}$.

Equation (42) above can be linked to an eddy break down process, which leads to energy dissipated by viscosity. If applied appropriately to structures transmitted through a "worm hole" from a prior to a present universe, it can explain

1) How there could be a break up of "encapsulating" structure which may initially suppress additional entropy beyond $S_{\text {initial }} \sim 10^{5}$, in the onset of inflation. 
2) Provide a "release" mechanism $\Delta S \approx \Delta N_{\text {gravitons }}<10^{54} \ll 10^{88}$, with $\Delta S \approx \Delta N_{\text {gravitons }} \sim 10^{21}$ perhaps a starting point for increase in entropy in $\Delta t \approx t_{\text {Planck }} \sim 5 \times 10^{-44} \mathrm{sec}$, rising to $\Delta S \approx \Delta N_{\text {gravitons }} \leq 10^{54} \ll 10^{88}$ for times up to 1000 seconds after the big bang.

Let us now consider the impact of the octonian gravity paradigm and where it may break down. And why.

\section{Finally, Relic Graviton Produced Entropy at the Onset of the Big Bang. Why Starting Entropy Would Be So Small While CMBR Entropy Would Be So Large}

As a closing remark, Beckwith wishes to suggest a solution to Penrose's implied question about entropy as raised in Edingborough, Scotland [27] conference proceedings. Penrose talks about the $2^{\text {nd }}$ law, and its implied requirements as to the small initial value of early universe entropy, and then states that gravitational entropy would not be so major, whereas CMBR matter contributed entropy would be much larger. Beckwith is convinced that relic graviton production at the onset of the big bang, i.e. before the contribution of entropy from matter itself would be necessary to boost entropy from its small $10^{5}$ value at the onset of the big bang, to a much higher level, and that entropy would be initially dramatically boosted by that process, i.e. the uniformity requirement Penrose talks about in structure would be actually as of up to the Electro weak transition, and far after the initial onset of inflation itself.

\section{A New Idea Extending Penrose's Suggestion of Cyclic Universes, Black Hole Evaporation, and the Embedding Structure Our Universe Is Contained Within}

We would have that Equation (42) in itself would mean that in the pre Planckian physics regime, and in between $\pm l_{p}$, QM no longer applies. What we will do next is to begin the process of determining a regime in which Equation (34) may no longer hold via experimental data sets. As an example of present confusion, please consider the following discussion where leading cosmologists, i.e. Sean Carroll [17] (2005) asserted that there is a distinct possibility that mega black holes in the center of spiral galaxies have more entropy, in a calculated sense, I.e. up to $10^{90}$ in non dimensional units. This has to be compared to Carroll's (2005) [17] stated value of up to $10^{88}$ in non dimensional units for observable non dimensional entropy units for the observable universe. Assume that there are over one billion spiral galaxies, with massive black holes in their center, each with entropy $10^{90}$, and then there is due to spiral galaxy entropy contributions $10^{6} \times 10^{90}=10^{96}$ entropy units to contend with, vs. $10^{88}$ entropy units to contend with for the observed universe, i.e. at least a ten to the eight order difference in entropy magnitude to contend with. A further datum to consider is that Equation (44) with its variance of density fluctuations may eventually be linkable to Kolmogrov theory as far as structure formation. If we look at R. M. S. Rosa [18] 
[19] (2006), and energy cascades of the form of the "energy dissipation law", assuming $u_{0}, l_{0}$ are minimum velocity and length, with velocity less than the speed of light, and the length at least as large, up to $10^{6}$ time larger than Planck length $l_{\text {Planck }}$

$$
\varepsilon \approx \frac{u_{0}^{3}}{l_{0}}
$$

Equation (43) above can be linked to an eddy break down process, which leads to energy dissipated by viscosity. If applied appropriately to structures transmitted through a "worm hole" from a prior to a present universe, it can explain

1) How there could be a break up of "encapsulating" structure which may initially suppress additional entropy beyond $S_{\text {initial }} \sim 10^{5}$, in the onset of inflation

2) Provide a "release" mechanism $\Delta S \approx \Delta N_{\text {gravitons }}<10^{54} \ll 10^{88}$, with $\Delta S \approx \Delta N_{\text {gravitons }} \sim 10^{21}$ perhaps a starting point for increase in entropy in $\Delta t \approx t_{\text {Planck }} \sim 5 \times 10^{-44} \mathrm{sec}$, rising to $\Delta S \approx \Delta N_{\text {gravitons }} \leq 10^{54} \ll 10^{88}$ for times up to 1000 seconds after the big bang.

Let us now consider the impact of the octonian gravity paradigm and where it may break down. And why.

\section{First Part of Conclusion: A New Idea Extending Penrose's Suggestion of Cyclic Universes, Black Hole Evaporation, and the Embedding Structure Our Universe Is Contained Within}

Beckwith strongly suspects that there are no fewer than $N$ (a large number) of universes undergoing Penrose "infinite expansion" and all these are contained within a mega universe structure. Furthermore, that each of the $N$ universes has black hole evaporation commencing, with the Hawking radiation from decaying black holes. If each of the $N$ universes is definable by a partition function, we can call $\left\{\Xi_{i}\right\}_{i \equiv N}^{i \equiv 1}$, then there exist an information minimum ensemble of mixed minimum information roughly correlated as about $10^{7}-10^{8}$ bits of information per each partition function in the set $\left.\left\{\Xi_{i}\right\}_{i \equiv N}^{i \equiv 1}\right|_{\text {before }}$, so minimum information is conserved between a set of partition functions per each universe

$$
\left.\left.\left\{\Xi_{i}\right\}_{i \equiv N}^{i \equiv 1}\right|_{\text {before }} \equiv\left\{\Xi_{i}\right\}_{i=N}^{i \equiv 1}\right|_{\text {after }}
$$

However, that there is non-uniqueness of information put into each partition function $\left\{\Xi_{i}\right\}_{i \equiv N}^{i \equiv 1}$. Furthermore that within the mega structure, that Hawking radiation from the black holes is collated via a strange attractor collection in the mega universe structure to form a new big bang for each of the $\mathrm{N}$ universes as represented by $\left\{\Xi_{i}\right\}_{i \equiv N}^{i \equiv 1}$. Verification of this mega structure compression and expansion of information with a non unique venue of information placed in each of the $\mathrm{N}$ universes would strongly favor Ergodic mixing treatments of initial values for each of the $\mathrm{N}$ universes expanding from a quasi singularity beginning. If this idea is in any way confirmable, it would lend credence as to the formation of the dark flow hypothesis, and of how a harmonic perturbative contri- 
butions to initial inflationary expansion may occur, within a partially random ergotic background. Beckwith claims that such a process would inherently favor the small $10^{7}$ bits of information per each partition function representing the "start" of expansion of a new universe. Hopefully, in doing so, one can explain, energy flux being re formulated for each universe. i.e. start with the Alcubierre's formalism about energy flux, assuming that there is a solid angle for energy distribution $\Omega$ for the energy flux to travel through [27], and [28].

$$
\frac{\mathrm{d} E}{\mathrm{~d} t}=[\lim r \rightarrow \infty]\left[\frac{r^{2}}{16 \pi}\right] \oint\left|\int_{-\infty}^{t} \Psi_{4} \mathrm{~d} t^{\prime}\right|^{2} \cdot \mathrm{d} \Omega
$$

The expression $\Psi_{4}$ is a Weyl scalar which we will, before the electro weak phase transition, assume that time dependence of both $h^{+}$and $h^{x}$ is miniscule and that initially $h^{+} \approx h^{x}$, so as to initiate $\Psi_{4}$ as

$$
\Psi_{4} \cong-\frac{1}{4} \cdot\left[+\partial_{r}^{2} h^{+}\right] \cdot(-1+i)
$$

The upshot, is that the initial energy flux about the inflationary regime would lead to looking at

$$
\left|\int_{-\infty}^{t} \Psi_{4} \mathrm{~d} t^{\prime}\right| \approx\left|\frac{1}{2} \cdot\left[+\partial_{r}^{2} h^{+}\right]\right| \cdot\left(\tilde{n} \cdot t_{\text {Planck }}\right)
$$

This will lead to an initial energy flux at the onset of inflation which will be presented as

$$
\frac{\mathrm{d} E}{\mathrm{~d} t}=\left[\frac{r^{2}}{64 \pi}\right] \cdot\left|+\partial_{r}^{2} h^{+}\right|^{2} \cdot\left[\tilde{n} \cdot t_{\text {Planck }}\right]^{2} \cdot \Omega
$$

If we are talking about an initial energy flux, we then can approximate the above as

$$
E_{\text {initial-flux }} \cong\left[\frac{r^{2}}{64 \pi}\right] \cdot\left|+\partial_{r}^{2} h^{+}\right|^{2} \cdot\left[\tilde{n} \cdot t_{\text {Planck }}\right]^{3} \cdot \Omega_{\text {effective }}
$$

Inputs into both the expression $\left|\partial_{r}^{2} h^{+}\right|$, as well as $\Omega_{\text {effective }}$ will comprise the rest of this document, plus our conclusions. The derived value of $\Omega_{\text {effective }}$ as well as $E_{\text {initial-flux }}$ will be tied into a way to present energy per graviton, as a way of obtaining $n_{f}$. The $n_{f}$ value so obtained, will be used to make a relationship, using Y. J. Ng's entropy [9] counting algorithm of roughly $S_{\text {entropy }} \sim n_{f}$. We assert that in order to obtain $S_{\text {entropy }} \sim n_{f}$ from initial graviton production, as a way to quantify $n_{f}$, that a small mass of the graviton can be assumed. How to tie in this energy expression, as given in Equation (49) will be to look at the formation of a non trivial gravitational measure which we can state as a new big bang for each of the $N$ universes as represented by [23], and [29] and $n\left(E_{i}\right)$ the density of states at a given energy $E_{i}$ for a partition function defined by

$$
\left\{\Xi_{i}\right\}_{i=1}^{i \equiv N} \propto\left\{\int_{0}^{\infty} \mathrm{d} E_{i} \cdot n\left(E_{i}\right) \cdot \mathrm{e}^{-E_{i}}\right\}_{i=1}^{i \equiv N} .
$$


Each of the terms $E_{i}$ would be identified with Equation (50) above, with the following iteration given, namely for $N$ universes

$$
\left.\left.\frac{1}{N} \cdot \sum_{j=1}^{N} \Xi_{j}\right|_{j \text {-before-nucleation-regime }} \stackrel{\text { vacuum-nucleation-tranfer }}{\longrightarrow} \Xi_{i}\right|_{i \text {-fixed-after-nucleation-regime }}
$$

For $N$ number of universes, with each $\left.\Xi_{j}\right|_{j \text {-before-nucleation-regime }}$ for $j=1$ to $N$ being the partition function of each universe just before the blend into the RHS of Equation (52) above for our present universe. Also, each of the independent universes given by $\left.\Xi_{j}\right|_{j \text {-before-nucleation-regime }}$ would be constructed by the absorption of one million black holes sucking in energy. i.e. in the end

$$
\left.\left.\Xi_{j}\right|_{j \text {-before-nucleation-regime }} \approx \sum_{k=1}^{M a x} \tilde{\Xi}_{k}\right|_{\text {black-holes-jth-universe }}
$$

One can treat Equation (52) as a de facto Ergodic mixing of prior universes to a present universe, with the partition function of each of the universes defined by Equation (51) above. Filling in the inputs into Equation (50) to Equation (52) is what will be done in the months ahead. See [29] as to what was done with this idea, in terms of the Penrose Cyclic conformal cosmology conjecture. Ergodic mixing as covered is in [30] is for the Mixing hypothesis whereas our multiverse generalization of the Penrose conjecture is a multiverse version of what he wrote up for single repeating universes as written in [31]. We also look forward to working in details as given by t'Hooft in [32] too.

\section{Conclusions: Several Reasons for the Analog Nature of Reality with Digital a Sub Set of a Larger Analog Basis}

We wish to summarize what we have presented in an orderly fashion. Doing so is a way of stating that Analog; reality is the driving force behind the evolution of inflationary physics:

1) Pre-Octonian gravity physics (analog regime of reality) features a breakdown of the Octonian gravity commutation relationships when one has curved space time. This corresponds to, as brought up in the Jacobi iterated mapping for the evolution of degrees of freedom to a buildup of temperature as thermal heat influx for an increase in degrees of freedom from 2 to over 1000, per unit volume of space time. The peak regime where the degrees of freedom maximize out is where the Octonian regime holds, corresponding to, also, Octonian gravity, when one has flat space, after a significant increase in temperature.

2) Analog physics, prior to the buildup of temperature can be represented by the mappings given by Equation (53) and Equation (54). The first of these mappings is an ergotic mapping, a perfect mixing regime from many universes into our own present universe. By necessity, this mapping requires a deterministic quantum limit as similar to what tHooft included in his embedding of Quantum physics in a larger, non-linear theory. This is approximated by current Pilot model buildup of an embedding of QM within a more elaborate super structure. 
3) The types of discontinuities presented, in Equation (42), Equation (22), Equation (14a), Equation (14b) are ways to the necessity of $\left|\frac{\eta}{s} \approx \varepsilon^{+}\right| \ll \frac{1}{4 \pi}$ giving only $\eta \neq 0, \varepsilon \rightarrow \infty$, instead of $\eta \rightarrow 0^{+}$, with the later case designating when entropy vanishes, which would correspond to no information from prior universes being transferred, i.e. non-zero viscosity corresponding to, with almost infinite energy, when the approach to Octonionic gravity occurs. The other case when viscosity vanishes would be tantamount to when no information is exchanged.

Understanding the nature of the ergotic mapping inherent in Equation (53) and Equation (54) would allow for a rigorous understanding of the necessity of $\left|\frac{\eta}{s} \approx \varepsilon^{+}\right| \ll \frac{1}{4 \pi}$ giving only $\eta \neq 0, \varepsilon \rightarrow \infty$, instead of $\eta \rightarrow 0^{+}$.

We hope that understanding these issues allows for determining how $\mathrm{K}$ essence physics can contribute to emergent structure, and perhaps massive gravitons and avoid symmetry breaking potentials, as used for the Higgs boson, so mentioned in Equation (29) and Equation (30). In doing so, we see first Analog physics in pre-Planckian space time, then, briefly the formation of Digital reality, as paramount in the beginning of inflationary cosmology. The genesis of this reality is from an analog physics foundation.

\section{Acknowledgements}

This work is supported in part by National Nature Science Foundation of China grant No. 11375279.

\section{Conflicts of Interest}

The authors declare no conflicts of interest regarding the publication of this paper.

\section{References}

[1] Beckwith, A.W. (2009) Relic High Frequency Gravitational Waves from the Big Bang, and How to Detect Them. AIP Conference Proceedings, 1103, 571. https://doi.org/10.1063/1.3115567

[2] Park, D.K., Kim, H. and Tamarayan, S. (2002) Nonvanishing Cosmological Constant of Flat Universe in Brane World Senarios. Physics Letters B, 535, 5-10. https://doi.org/10.1016/S0370-2693(02)01729-X

[3] Barvinsky, A., Kamenschick, A. and Yu, A. (2006) Thermodynamics from Nothing: Limiting the Cosmological Constant Landscape. Physical Review D, 74, Article ID: 121502 (Rapid Communications). https://doi.org/10.1103/PhysRevD.74.121502

[4] Beckwith, A.W. and Beckwith, A.W. (2008) Implications for the Cosmological Landscape: Can Thermal Inputs from a Prior Universe Account for Relic Graviton Production? AIP Conference Proceedings, 969, 1091-1102. https://doi.org/10.1063/1.2844947

[5] Radu, E. and Tchrakian, D. (2009) Gravitating Yang-Mills Fields in All Dimensions. https://arxiv.org/abs/0907.1452 
[6] Frampton, P.H. and Baum, L. (2007) Turnaround in Cyclic Cosmology. Physical Review Letters, 98, Article ID: 071301.

[7] Hooft, G. (2010) The Emergence of Quantum Mechanics. Frontiers of Fundamental Physics, Paris, FFP11.

[8] Perkins, D.H. (2005) Particle Astrophysics, Oxford Master Series in Particle Physics. Oxford University Press, Oxford.

[9] Ng, Y.J. (2008) Article: Spacetime Foam: From Entropy and Holography to Infinite Statistics and Nonlocality. Entropy, 10, 441-461. https://doi.org/10.3390/e10040441

[10] Glinka, L. (2007) Quantum Information from Graviton-Matter Gas. Sigma, 3, 087. https://doi.org/10.3842/SIGMA.2007.087

[11] Goldberger, W.D. (2007) Effective Field Theories and Gravitational Radiation. In: Bernardeau, F., Grogean, C. and Dalibard, J., Eds., Particle Physics and Cosmology, the Fabric of Space Time, Elsevier, Oxford, 351-396.

[12] Asakawa, M., Hatsuda, T. and. Nakahara, Y. (2001) Maximum Entropy Analysis of the Spectral Functions in Lattice QCD. Progress in Particle and Nuclear Physics, 46, 459-508. https://doi.org/10.1016/S0146-6410(01)00150-8

[13] Asakawa, M., Bass, S.A. and Müller, B. (2006) Anomalous Viscosity of an Expanding Quark-Gluon Plasma. Physical Review Letters, 96, Article ID: 252301. https://doi.org/10.1103/PhysRevLett.96.252301

[14] Torrieri, G. and Mishustin, I. (2008) Instability of Boost-Invariant Hydrodynamics with a QCD Inspired Bulk Viscosity. Physical Review Letters, 78, Atticle ID: 021901. http://arxiv.org/abs/0805.0442 https://doi.org/10.1103/PhysRevC.78.021901

[15] Weinberg, S. (2008) Cosmology. Oxford University Press, New York.

[16] Beckwith, A.W., Li, F.Y., et al. (2011) Is Octonian Gravity Relevant near the Planck Scale? If Gravity Waves Are Generated by Changes in the Geometry of the Early Universe, How Can We Measure Them? http://vixra.org/abs/1101.0017

[17] Beckwith, A.W. (2011) How to Use the Cosmological Schwinger Principle for Energy Flux, Entropy, and "Atoms of Space Time", for Creating a Thermodynamics Treatment of Space-Time. http://iopscience.iop.org/article/10.1088/1742-6596/306/1/012064

[18] Lynch, S. (2007) Dynamical Systems with Applications Using Mathematica. Birkhäuser, Boston.

[19] Sarkar, U. (2008) Particle and Astroparticle Physics, Series in High Energy Physics, Taylor and Francis. Boca Racon, Florida.

[20] Beckwith, A.W. (2011) Entropy Production and a Toy Model as to Irregularities in the CMBR Spectrum. http://vixra.org/abs/1102.0007

[21] Scherrer, R.J. (2004) Purely Kinetic k-Essence as Unified Dark Matter. Physical Review Letters, 93, Article ID: 011301. https://doi.org/10.1103/PhysRevLett.93.011301

[22] Crowell, L. (2005) Quantum Fluctuations of Spacetime. In: World Scientific Series in Contempoary Chemical Physics, Vol. 25, World Scientific Publishing Co. Singapore, Singapore. https://doi.org/10.1142/5952

[23] Poplawski, N. (2011) Cosmological Constant from QCD Vacuum and Torsion. Annalen der Physik, 523, 291-295. https://doi.org/10.1002/andp.201000162

[24] Caroll, S. (2014) Spacetime and Geometry: An Introduction to General Relativity. Pearson Education Ltd., London. 
[25] Foias, C., Manley, O.P., Rosa, R.M.S. and Temam, R. (2001) Estimates for the Energy Cascade in Three-Dimensionalturbulent. Comptes Rendus de I'Académie des Sciences Series I: Mathematics, 333, 499. https://doi.org/10.1016/S0764-4442(01)02008-0

[26] Rosa, R.M.S. (2006) Turbulence Theories. In: Franchioise, J.-P., Naber, G.L. and Tsun, T.S., Eds., Encyclopedia of Mathematical Physics, Vol. 2, Elsevier, Geneva, 253-261.

[27] Penrose, R. (2006) Before the Big Bang: An Outrageous New Perspective and Its Implications for Particle Physics. Proceedings of EPAC 2006, 26-30 June 2006, Edinburgh, Scotland, 2759-2763.

[28] Alcubierre, M. (2008) Introduction to $3+1$ Numerical Relativity. Oxford University Press, New York. https://doi.org/10.1093/acprof:oso/9780199205677.001.0001

[29] Beckwith, A.W. (2014) Analyzing Black Hole Super-Radiance Emission of Particles/Energy from a Black Hole as a Gedankenexperiment to Get Bounds on the Mass of a Graviton. http://www.hindawi.com/journals/ahep/2014/230713/

[30] Dye, H.A. (1965) On the Ergodic Mixing Theorem. Transactions of the American Mathematical Society, 118, 123-130. https://doi.org/10.1090/S0002-9947-1965-0174705-8

[31] Penrose, R. (2011) Cycles of Time-An Extrardinary New View of the Universe, Alfred A. Knopf, New York.

[32] Hooft, G.T. (2002) Determinism beneath Quantum Mechanics. https://arxiv.org/abs/quant-ph/0212095 